Also of concern is the lack of evaluation of harm to patients caused by what is essentially a screening programme of high-risk individuals. Such programmes are known to be associated with harm in a variety of forms. These include overdiagnosis, overtreatment and anxiety concerning the illness being investigated. ${ }^{4}$

Last, for a patient to give informed consent to participate in this kind of programme, they should be informed of the uncertainties inherent in it and the likelihood or otherwise of benefit to them of such a screening.

It is time to take stock and critically review which, if any, of these investigations are necessary for our patients.

1 Gumber R, Abbas M, Minajagi M. Monitoring the metabolic sideeffects of atypical antipsychotics. Psychiatrist 2010; 34: 390-5.

2 Osborn DP, Levy G, Nazareth I, Petersen I, Islam A, King MB. Relative risk of cardiovascular and cancer mortality in people with severe mental illness from the United Kingdom's General Practice Research Database. Arch Gen Psychiatry 2007; 64: 242-9.

3 Fourth Joint Task Force of the European Society of Cardiology and Other Societies on Cardiovascular Disease Prevention in Clinical Practice. European guidelines on cardiovascular disease prevention in clinical practice: executive summary. Eur J Cardiovasc Prevent Rehabil 2007; 14 (suppl 2): E1-40.

4 Jørgensen K, Gøtzsche P. Content of invitations for publicly funded screening: mammography. BMJ 2006; 332: 538-41.

Paul F. Reed is Consultant Psychiatrist with Lancashire Care NHS Foundation Trust, email: paul.reed@lancashirecare.nhs.uk

doi: 10.1192/pb.34.12.540a

\section{Scarcity of evidence base on management of acutely disturbed patients}

Brown et al give a useful insight into the practice at seven intensive care units all over the country. ${ }^{1}$

Their results show that $22 \%$ of patients were given rapid tranquillisation using the intramuscular route and $68 \%$ were not given any rapid tranquillisation medication at all.

The results table is confusing and the numbers do not add up; $3 \%$ appear not to have been given any medication at all, which causes concerns about the referral process to psychiatric intensive care units (PICUs) and whether patients were appropriately placed.

The study does not clarify the legal status of the patients and does not throw any light on the level of aggression of the patients in PICUs.

The most common diagnosis was schizophrenia/schizoaffective disorder (54\%), followed by mania (19\%) and substance misuse ( $8 \%$ ). The diagnosis for $19 \%$ of patients has not been provided in the study.

1 Brown S, Chhina N, Dye S. Use of psychotropic medication in seven English psychiatric intensive care units. Psychiatrist 2010; 34: 130-5.

Rupali Acharya CT2 Psychiatry, Trafford, Manchester, email: rupa_acharya@hotmail.com, Khurram Tanveer Sadiq Locum Consultant Psychiatrist, Trafford, Manchester

doi: $10.1192 / \mathrm{pb} .34 .12 .541$

\section{Authors' reply}

We are keen to encourage a wider discussion of the issues around the treatment of patients admitted to psychiatric intensive care units (PICUs) and welcome the opportunity to address points raised by Acharya \& Sadiq. In writing the paper ${ }^{1}$ we made a series of judgements about how best to present a large volume of data in an easily assimilated form and we are sorry if some of these decisions led to a lack of clarity.

One of the main findings of the study was that most PICU patients are safely managed without recourse to forced intramuscular (IM) medication, indeed that some patients are managed without any psychotropic medication at all. The study only collected data about treatment while the patients were in a PICU (this was a pragmatic decision as many patients came from and returned to distant units where data collection was not feasible). We suspect that some of the patients who did not receive any psychotropic medication in the PICU had received medication before transfer, possibly in the form of medium- or long-acting antipsychotic injection. Others will have received medication after transfer to the acute ward. The diagnoses of those patients who did not receive any medication were: schizophrenia (1), depression (2), druginduced psychosis (1), substance dependence (2), personality disorder (2), anxiety (1) and adjustment disorder (1).

The numbers in Table 1 do not always add up to $100 \%$ because some patients appear in several categories, for example: they were given IM rapid tranquillisation and IM zuclopenthixol acetate. All figures were rounded to the nearest $0.5 \%$; with this caveat we are confident that the appropriate figures (from text and table) do add up to $100 \%$.

The primary diagnoses of patients aggregated into the category 'other' were: learning (intellectual) disability, dementia, Asperger syndrome, obsessive-compulsive disorder, anxiety, adjustment disorder, and intoxication with drugs or alcohol.

We address the legal status of the patients and the level of behavioural disturbance more fully in a companion paper. ${ }^{2}$ With respect to the legal status of the patients, the findings were: 10 informal (3\%), 7 on Section 5(2) (2\%), 123 Section 2 (37\%), 158 Section 3 (48\%), 1 Section $4(<1 \%)$, 9 Section 37 (3\%), 19 a range of forensic sections covering different transfers from prison (6\%).

With respect to measurement of behavioural disturbance and mental state, we used the Brief Psychiatric Rating Scale (BPRS) and those subscales (hostility score, three-item Factor $\checkmark$ cluster and five-item hostility cluster) which focus on behavioural disturbance. The mean BPRS score fell from 58.2 on admission to 39.8 on transfer from PICU; the respective figures for the hostility score, Factor $V$ and hostility clusters were: 4.2 to $1.8,9.2$ to 5.5 and 17.3 to 11.1 .

We hope that these details clarify the points raised by Acharya \& Sadiq.

1 Brown S, Chhina N, Dye S. Use of psychotropic medication in seven English psychiatric intensive care units. Psychiatrist 2010; 34: 130-5.

2 Brown S, Chhina N, Dye S. The psychiatric intensive care unit: a prospective survey of patient demographics and outcomes at seven English PICUs. J Psychiatr Intensive Care 2008; 4: 17-27.

Steve Brown is Consultant Psychiatrist, Hampshire Partnership NHS Trust, email: Steve.Brown@hantspt-sw.nhs.uk

doi: $10.1192 / p b .34 .12 .541 a$

\section{Comment on the evaluation of the Time to Change anti-stigma campaign}

The study by Abraham et al suggests that a single exposure to selected Time to Change campaign material (those including the ' 1 in 4 ' message) delivered via post was not effective at improving attitudes towards people with mental illness. 